\title{
Los centros de incubación de negocios y la gestión de redes: exploración empírica aplicando el análisis de redes sociales
}

\author{
Noel M. Muñiz \\ Alfonso C. Morales-Gutiérrez \\ J. Antonio Ariza-Montes \\ Universidad de Córdoba \\ noelmunic@yahoo.com; ariza@etea.com
}

Resumen: Las incubadoras de negocios consideran la gestión de redes como un elemento crítico para generar capacidades competitivas; el modelo más evolucionado actualmente es conocido como «incubación en red». Sin embargo, diversos investigadores aducen la necesidad de relacionar estos dos constructos positivamente. El estudio explora la aplicación de la «teoría de redes» al campo del emprendimiento a través de las interconexiones, entre incubados y grupos de interés, y las magnitudes propias de dicha teoría. La muestra comprende 18 empresas asistidas bajo la modalidad de «acompañamiento externo». Su originalidad radica en explorar las claves del éxito de la gestión de incubación desde la perspectiva del análisis de redes. Por último, se aporta una serie de recomendaciones para futuros estudios.

Palabras clave: creación de negocios, incubación de empresas, networking, análisis de redes, competitividad.

Abstract: Business incubators consider network management as fundamental to the generation of competitive capabilities: currently the most evolving model is known as networked incubation. However, many researchers argue that these two constructs need to be positively related. The study explores the application of Social Network Theory to the field of entrepreneurship through interconnections between incubatees

* In memoriam. 
and stakeholders, and the typical magnitudes of the theory. The sample comprises 18 endeavors that had the assistance of external support. The originality of the study lies in the fact that it explores the keys to the successful management of incubation from the perspective of Social Network Analysis. Finally, it provides a series of recommendations for further studies.

Keywords: business creation, business incubation, networking, social network analysis, competitiveness. 


\section{Planteamiento del problema: introducción al tema en estudio}

El fomento del desarrollo de emprendimientos en espacios protegidos de la gestión empresarial tiene como principales herramientas de promoción las incubadoras o viveros de empresas. Estos entornos centran su actividad en facilitar medios y promover servicios relevantes para el desempeño de los negocios que están siendo incubados: salas de trabajo y reunión, equipos de oficina y servicios relacionados con nuevas tecnologías. Asimismo, prestan apoyo en la fase de constitución del negocio, en la obtención de fondos financieros, en materia de innovación y conocimiento y, en relación con el tema de este trabajo, en el desarrollo de redes de contactos o networking.

Desde su concepción inicial de entidades facilitadoras de espacios de trabajo y servicios compartidos — década de 1970—, la gestión de la incubación ha evolucionado hacia una conceptualización más compleja e inspiradora del talento empresarial y humano, integrando en sus procesos la necesidad constante de desarrollar conocimiento, innovación, nuevas tecnologías y redes relacionales de trabajo (Hansen et alii, 2000).

Quizás por ello, el nivel organizacional más avanzado en la gestión de la incubación se fundamenta en una intensa generación de capital social - sinergias y economías de alcance en cooperación- gestado y autogenerado de forma activa por sus participantes (Hughes et alii, 2007). Estas conexiones se multiplican de forma exponencial una vez que las incubadoras deciden compartir habilidades más allá de las fronteras físicas. Precisamente, la apertura al exterior es la que ha acelerado los procesos de innovación y conocimiento, dando origen al nacimiento de una noción renovada de la incubación de emprendimientos: las incubadoras en red, también conocidas como networked incubators (Hansen et alii, 2000).

Por tanto, las redes relacionales de trabajo y su gestión (networking), fundamentada en el uso intensivo de prácticas de generación de capital social y de cooperación, constituyen hoy día pilares fundamentales de los principios del desarrollo de emprendimientos y su competitividad (Hughes et alii, 2007). Este salto organizacional ofrece nuevas posibilidades en la búsqueda de ventajas competitivas, especialmente para las pequeñas y medianas empresas desarrolladas en estos espacios protegidos. A pesar de que cada vez más los viveros de empresas adaptan sus organizaciones al trabajo en red, pareciendo confirmar el desarrollo evolutivo hacia el modelo de las incubadoras en red, aún resulta necesaria mayor investigación científica dirigida a explorar las relaciones que existen entre el networking y los factores clave de la competitividad empresarial (Bøllingtoft y Ulhøi, 2005). 


\section{Objeto de estudio}

La presente investigación tiene como objetivo principal explorar la aplicabilidad del análisis de redes a la gestión de incubación de empresas y, por tanto, la posibilidad de obtener datos cuantificables con el empleo de medidas relacionales propias de la teoría de redes: medidas de centralidad, densidad, etc.

A través de la observación de un vivero de empresas de carácter público - que en la actualidad conduce su propio programa de incubación bajo la modalidad de coaching externo-, se estudian las características de los vínculos relacionales que existen entre los incubados y sus principales grupos de interés.

De los resultados de este análisis se extraen conclusiones y recomendaciones de gran utilidad para comprender la relación existente entre la gestión de redes, los procesos de incubación empresarial y la generación de capacidades competitivas.

\section{Fundamentación teórica}

\subsection{Los esquemas de incubación de empresas y sus procesos}

La primera incubadora de empresas como tal se estableció en 1959 en la ciudad de Batavia, condado de Genesee (Nueva York). Con el fin de albergar pequeñas empresas y ayudarlas a crecer desde sus inicios, el empresario Carlos Mancuso alquiló una gran nave en el centro industrial de Batavia. Pocos años después, los viveros de empresas se fueron extendiendo por todo el mundo: primero por Europa - Inglaterra y Francia- y luego al resto de los continentes (Albert y Gaynor, 2001).

Según la National Business Incubation Association de Estados Unidos (NBIA), en el año 2006 existían cerca de 7.000 incubadoras diseminadas por todo el mundo, de las cuales un 16\% se encontraba en Estados Unidos (Knopp, 2007). Las nuevas tecnologías han sido, en muy buena parte, responsables de este progresivo crecimiento.

Respecto a Europa, la mayoría de las incubadoras, que a fecha de 2012 ascendían a 1.100 unidades gestoras aproximadamente, suelen especializarse en un sector productivo concreto o en un número limitado de ellos, siendo el sector con mayor auge el de las tecnologías de la información y la comunicación (TIC): tres cuartas partes de los incubados operan a día de hoy en esta industria (Comisión Europea, 2002). 
Los emprendimientos basados en el uso intensivo de conocimiento conforman el segundo mayor número de unidades económicas asistidas en estos viveros: nuevas industrias - tales como el comercio electrónico y los servicios relacionados entre negocios business to business (B2B) -, emprendimientos orientados a la investigación y el desarrollo $(\mathrm{I}+\mathrm{D})$ y las empresas de tipo financiero-informativo. Menor representatividad numérica tienen las unidades económicas orientadas a la gestión de ventas, comercialización y distribución, al igual que aquellas pertenecientes al sector agropecuario (Comisión Europea, 2002).

Las empresas asistidas por los viveros de emprendimientos en el viejo continente tienen una amplia variedad de servicios a su disposición. Según un estudio conducido por Aerts et alii (2007) sobre una muestra observada de 107 incubadoras, en su gran mayoría las incubadoras están equipadas con salas de trabajo, de reuniones o de conferencias (96\%), promueven con intensidad la gestión de redes —networking - (88\%), ayudan a sus acompañados en la elaboración de los planes de negocios y en el desarrollo de sus ideas empresariales (86\%), contribuyen a la obtención de fondos (79\%), facilitan los equipos necesarios (77\%), y brindan consejos y recomendaciones en materia de marketing $(73 \%)$ y asesoramiento financiero (72\%), entre otros muchos. De entre todos estos servicios ofrecidos por las incubadoras de negocios, el networking está adquiriendo un papel fundamental.

Son muchos los autores que destacan la importancia del networking para garantizar el éxito de la creación de empresas (Freel, 2003; Tse, 2002; Hansen et alii, 2000; Johannisson, 1988). Los emprendedores, a través del empleo intensivo de estas redes, reducen los costes de transacción y de información, ya que tienen la posibilidad de compartir algunos recursos con otros emprendedores (Williamson, 1975). Por otra parte, la gestión de redes permite a los empresarios de nueva creación lograr economías de alcance derivadas de la utilización y distribución conjunta de insumos, servicios y otros bienes (Bailey y Friedlander, 1982; Panzar y Willig, 1981). En este sentido Ford (1998) enfatiza la importancia de la red de contactos como herramienta que permite aprovechar plenamente las tecnologías y transformarlas en soluciones para los mercados. Sin embargo, para comprender cómo el networking ha llegado a ser un instrumento vital para la gestión de emprendimientos en incubadoras de negocios, se debe conocer cómo han evolucionado los enfoques estratégicos en el seno de estas organizaciones. 
A) Los enfoques estratégicos propuestos por Smilor y Gill: de la rebabilitación
de edificios al crecimiento continuo y planificado de los emprendimientos. Smilor y Gill (1986) establecen, como posible clasificación inicial para la gestión de incubación, dos enfoques estratégicos basados en el manejo de las operaciones y de sus objetivos organizacionales. El primer enfoque, empleado en la década de 1970 y principios de la de 1980, se centra en la rehabilitación de edificios antiguos o vacantes y el arrendamiento de espacios adecuados y relativamente baratos para la gerencia de negocios. Esta estrategia se focaliza fundamentalmente en proveer de entornos físicos convenientes y no en gestar y desarrollar las propias ideas emprendedoras. La segunda estrategia se basa en un intento más consciente de procurar la aparición de nuevas unidades económicas y su crecimiento continuo.

B) La propuesta de Allen y McCluskey en el continuo evolutivo de los enfoques de la gestión de incubación: las incubadoras de gestión privada, académica, sin fines de lucro y de desarrollo tecnológico.

Allen y McCluskey (1990) proponen una estructura que aporte continuidad al enfoque estratégico evolutivo de la gestión de incubación de Smilor y Gill. Esta estructura se fundamenta en cuatro tipos organizacionales diferentes para la gestión de la incubación. El primer modelo es el de las incubadoras de gestión privada con capital semilla y fines lucrativos: organizaciones que hacen aportes económicos a sus incubados con el fin de obtener beneficios futuros. Estas incubadoras se caracterizarían por contar con patrocinio privado y porque su objetivo principal no es el de contribuir a la sociedad y a su problemática, sino el de obtener una rentabilidad económica para sus inversores. El segundo modelo organizacional es el de las incubadoras académicas — también llamadas parques de ciencia, parques de investigación o parques tecnológicos - fuertemente relacionadas, por tanto, con las universidades: su principal objetivo es la transferencia de conocimiento como producto de la investigación y la obtención de resultados en el desarrollo de nuevos bienes, servicios y tecnologías. El tercer modelo está constituido por las incubadoras de desarrollo sin ánimo de lucro patrocinadas por instituciones de gobierno o entes privados que buscan fomentar el crecimiento empresarial en la zona de incidencia. La última propuesta es muy parecida a la inicial, pues hace referencia a aquellas incubadoras de desarrollo tecnológico con fines lucrativos que básicamente buscan crear oportunidades de inversión y de transferencia de tecnología a través de sus incubados, obteniendo un considerable rédito como producto de su gestión. La diferencia con el primer modelo radica en que este 
tipo de incubadora actúa más como una incubadora privada independiente que como una incubadora privada corporativa (Allen y McCluskey, 1990).

C) El enfoque de Bollingtoft y Ulhoi en la gestión de incubación: las incubadoras de carácter corporativo y la incubación en red: networked incubation.

Bollingtoft y Ulhoi (2005) proponen agregar dos modelos organizacionales más a este continuo clasificatorio: las incubadoras corporativas y las incubadoras en red o networked incubators. Las primeras se caracterizan por el uso intensivo de recursos, y son desarrolladas y controladas por grandes empresas que buscan obtener nuevos productos, servicios o unidades económicas difusoras de nuevas experiencias, conocimientos y tecnologías: las conocidas como spin-off incubators. Por su parte, las incubadoras en red constituyen el último eslabón evolutivo en el desarrollo de organizaciones gestoras de emprendimientos y son, sin lugar a dudas, las de mayor interés para la presente investigación al caracterizarse por una gestión intensiva del networking.

\subsection{La gestión intensiva de networking en el acompañamiento de incubados (coaching) : la incubación en red}

Las definiciones más recientes de incubación de empresas enfatizan la necesidad de formalizar, en la gestión de acompañamiento o coaching de los incubados, las interconexiones constantes de diversas índoles y múltiples participantes. Según estos razonamientos, el proceso de incubación de un emprendimiento mejora cuando la empresa naciente se encuentra acompañada por una incubadora que hace uso intensivo de los vínculos relacionales. Por esta razón, la organización actual de la gestión de incubación es considerada como un proceso que facilita a sus emprendimientos asistidos la multiplicación de la creación de valor mediante la intensa participación en sistemas interconectados -interna y externamente, en red- y que, por lo tanto, proporciona amplias relaciones de negocios (Hansen et alii, 2000).

Hackett y Dilts (2004) definen los nuevos modelos de incubación como sistemas estratégicos de intervención y acompañamiento para emprendedores, orientados a agregar valor a la actividad de las empresas acompañadas dentro de un contexto provocado de múltiples interconexiones; por consiguiente, el equipo de gestión de un vivero de empresas tiene, dentro de sus funciones torales, la responsabilidad de crear las condiciones adecuadas para que dichas redes de contactos surjan, crezcan y se reproduzcan, quedando a disposición de los emprendimientos en proceso de incubación. A esta actividad integral, que relaciona la gestión 
intensiva de redes o networking con el acompañamiento o coaching en el contexto de los procesos de incubación empresarial, es a lo que se denomina networked incubators o incubadoras en red.

La característica distintiva de un vivero de empresas enfocado a la incubación en red es contar con mecanismos que fomenten las asociaciones entre equipos de emprendimientos orientados a la optimización de recursos y generación de conocimiento, facilitando así el flujo de ideas y talento entre las empresas (Hansen et alii, 2000). De esta manera las unidades económicas que están siendo acompañadas pueden interrelacionarse, interna o externamente, para obtener recursos y asociarse con otros agentes económicos a la velocidad de las demandas del mercado y de los competidores. Esta gestión, en los procesos de incubación, brinda a sus incubados el acceso preferencial a socios potenciales y a asesores.

Se podría decir que las incubadoras de empresas, debidamente diseñadas para la gestión en red, combinan lo mejor de dos mundos que, a simple vista, parecen incompatibles: la escala y el alcance económico de las grandes corporaciones y el espíritu emprendedor de las pequeñas empresas. Pero si la gestión de incubación en red ofrece estas ventajas tan convincentes ¿por qué no surge hasta ahora? La respuesta refleja un sutil aspecto de la economía contemporánea.

Las incubadoras de empresas en red aparecen muy recientemente en el contexto económico debido a la extrema necesidad - hoy mayor que nunca- de tener acceso a información, a recursos, a conocimientos, a técnicas, a fuentes financieras y a un interminable etcétera de insumos que aceleran la creación de valor y la generación de ventajas competitivas que solo trabajando «en red» se vuelven gestionables. Este método pone a disposición de sus incubados todas las condiciones necesarias para el desarrollo de asociaciones estratégicas valiosas, el reclutamiento de personal con talento y el acceso a expertos externos. Es por ello que se podría decir que existen dos características básicas de la gestión de incubación en red. En primer lugar, la creación y gestión de redes debe ser parte de un proceso formalmente institucionalizado; lo que significa que el vivero de empresas debe disponer de mecanismos intrínsecos que promuevan activamente las interconexiones múltiples. En segundo lugar, las incubadoras en red deben enfocarse en fomentar las interrelaciones que permitan tener acceso preferencial a contactos y no a trato preferencial de parte de estos (Hansen et alii, 2000). En otras palabras, las interrelaciones que provoque la gestión de incubación deben basarse en la percepción de que los intercambios realizados son justos, equilibrados y que responden a necesidades reales de las partes involucradas. Sin embargo, en la tarea de formalizar un proceso intensivo de gestión de redes, este tipo de incubadoras debe implementar dos prácticas esenciales. 
La primera práctica radica en diseñar una cartera estratégica óptima de contactos que puedan aprovechar los incubados. El segundo paso se basa en desarrollar el perfil de la red. El objetivo de este último procedimiento es gestionar la implementación de mecanismos que contribuyan a formalizar la creación y gestión actual y futura de la misma red; es decir, establecer conexiones y relaciones que permanezcan ancladas con mayor fuerza a la incubadora y, en menor medida, a los individuos o emprendimientos en particular. Algunas prácticas para alcanzar este objetivo son:

- La creación de vínculos formales con expertos externos.

- La convivencia y acompañamiento empresarial de expertos externos a los incubados.

- El establecimiento de reuniones ocasionales, pero con regularidad.

- La instauración de procesos de intercambio de información y conocimiento entre las empresas.

- La implementación de incentivos económicos.

- La contratación de agentes de mercado especializados en el cierre de acuerdos.

En un esfuerzo por establecer una tipología de los actuales esquemas de la gestión de incubación en red, Etzkowitz (2002) categoriza estas incubadoras de acuerdo con la dinámica de sus conexiones:

- Intrared: aquellas incubadoras en red que operan a través de redes internas entre las unidades económicas que la conforman.

- Interred: incubadoras en red que operan a través de conexiones externas entre incubadoras y empresas de diferentes viveros de empresas.

+ Extrared: aquellas operan a través de la formación de nuevas organizaciones creadas en el seno en las mismas incubadoras y que provienen de entidades económicas heterogéneas.

Por lo tanto, es de suponer que diferentes combinaciones de intercambio $y$ de uso en común de recursos, bajo dinámicas de conexiones distintas, generarán resultados distintos en incubadoras que promuevan la gestión de redes. Según Hughes et alii (2007) los cuatro tipos de resultados que pueden obtener los procesos de incubación en red son los siguientes: incubación hacia dentro, incubación especializada, incubación en comunidad e incubación dinámica. A continuación se detallan sus características:

- La incubación hacia dentro se produce cuando una organización gestora de emprendimientos pone a disposición de los miembros partícipes del proceso de interrelación - internos o externos-, una escasa cantidad de recursos que compartir; mostrando un bajo compromiso con el desarrollo estratégico de las redes de contactos. 
- La incubación especializada surge como resultado del compromiso de poner a disposición de los miembros partícipes del proceso de interrelación -internos o externos-, amplias concentraciones de recursos y limitadas interacciones basadas en la generación de conocimiento, así como en una estrategia sostenida de participación en red.

- La incubación en comunidad se manifiesta cuando una empresa, que está siendo incubada, se compromete a participar amplia y estratégicamente en la búsqueda de conocimiento, pero que colabora escasamente en la puesta en común de recursos.

- Por último, la incubación dinámica representa una actividad extensa de concentración de recursos en conjunción con una activa búsqueda de conocimiento a través del desarrollo de redes. Este sería el resultado más favorable al que puede aspirar una incubadora en el cumplimiento de su misión.

Lo verdaderamente promisorio de la gestión de incubación en red radica en su potencial para superar las actuales estructuras organizacionales que crean y desarrollan nuevas ideas y negocios. Aquellos modelos organizacionales que logren potencializar el dinamismo emprendedor y el acceso a redes de contactos, preservando los beneficios de economías de escala y de alcance, serán los más eficientes para alcanzar el éxito a largo plazo en el contexto de la nueva economía (Hansen et alii, 2000). La incubación de empresas en red es, pues, una de estas revolucionarias formas organizativas emergentes de trabajo, y en la actualidad se manifiesta bajo diferentes esquemas y tipos.

\subsection{La teoría redes en el contexto de las organizaciones y la creación de valor}

Parte importante de la base teórica de este documento la constituye la teoría de redes o del capital social, cuyos términos son utilizados indistintamente en la literatura económica (Bollingtoft y Ulhoi, 2005). Esta teoría se fundamenta en el hecho de que toda relación, más allá de lo contractual y puramente económico, es impulsada por importantes dimensiones sociales que deben considerarse al analizar el mencionado vínculo relacional.

La gestión de redes genera capital social, entendido este como un constructo de recursos que, siendo propios de una estructura social cualquiera, se activa y se hace accesible debido a acciones intencionales y programadas (Lin, 2001). Estas interacciones, según Bolino et alii (2002), contribuyen a que los individuos logren trabajar, en conjunto, con mayor eficacia y eficiencia cuando se conocen entre sí, se tienen confianza y se identifican recíprocamente. El capital social, por tanto, 
está compuesto por redes sociales individuales y colectivas, lazos y estructuras que ofrecen al individuo acceso a información y conocimiento (Bollingtoft y Ulhoi, 2005). Es por ello por lo que el emprendedor debe entrar en contacto con otros individuos que puedan aportar nuevas ideas y recursos complementarios (Larson, 1991; Johannisson, 1988).

En el contexto de los procesos de incubación empresarial y la gestión de networking, las redes generadas internamente resultan particularmente útiles para la creación de capital social debido a que permiten, a un conjunto de unidades económicas diversas, compartir todo tipo de recursos (Lyons, 2000). Este autor considera que el servicio más importante ofrecido en la actualidad por las incubadoras es la oportunidad de crear redes entre empresas acompañadas; es decir, la gestión de redes a nivel interno de la organización. Por su parte, las redes externas se convierten en cruciales para los incubados ya que los vinculan y los ponen en contacto con potenciales socios, clientes, empresas locales, etc., más allá de los límites de su propia red.

El hecho de gestionar ambos tipos de redes estratégicamente genera réditos directos para los incubados. Según Uzzi (1996) la teoría del capital social demuestra que los emprendimientos capaces de establecer sólidas conexiones en red pueden llegar a disfrutar de ventajas significativas en el cumplimiento de sus metas organizacionales. El hecho de que los incubados convivan bajo un mismo techo eleva la probabilidad de colaboración entre ellos y, por tanto, la posibilidad del cumplimiento de sus objetivos individuales y colectivos.

Sin embargo, Bollingtoft y Ulhoi (2005) sostienen que aún queda mucho por conocer sobre los mecanismos que facilitan u obstaculizan la creación de redes internas o externas y, por consiguiente, de capital social en la gestión de networking. Tampoco se conoce mucho sobre la naturaleza de estas redes. En lo que sí coincide plenamente la comunidad científica es en que para enfrentar la competitividad del mercado actual no se puede actuar de forma aislada e individual en la búsqueda de ventajas competitivas.

El límite que las empresas tienen para crecer y competir se reduce a sus propias capacidades en materia de recursos y conocimientos. Dada la escasez con que se presentan dichos factores, los emprendimientos nacientes muestran un desempeño relativamente débil y tienden a crecer y desarrollarse de manera inestable. Hughes et alii (2007) consideran que la colaboración hace más visibles a las empresas y les permite gestionar, a través de asociaciones basadas en el intercambio de recursos y conocimientos, un mayor número de logros en conjunto que trabajando por separado. La evidencia sugiere que el desarrollo de capital social, a través de la gestión del networking y el activo aprovechamiento de las oportu- 
nidades que brinda la creación de redes, representa una vía de acceso a ventajas competitivas para las empresas (Ireland et alii, 2000).

Cuando una o varias unidades económicas desarrollan y mantienen relaciones interactivas con otros emprendimientos, las oportunidades de creación de valor surgen del capital social generado por dichas aproximaciones entre empresas y se multiplican a medida que dichas interconexiones se profundizan (Coleman, 1988). En esencia, el capital social representa un constructo de buenas voluntades, una credencial de principios que es desarrollada por un actor a través de sus acciones en el tiempo y que contribuye a la creación de valor incidiendo en las condiciones necesarias para que este se produzca (Burt, 1992).

Esta dimensión relacional del capital social, que nace de la misma interacción social y que representa activos creados, compartidos y optimizados, es consistente con la acción de búsqueda continua de ventajas competitivas (Dyer y Singh, 1998). Dichos activos son recursos reales y potenciales que las organizaciones conservan latentes en su interior y de los que disponen como producto de las interrelaciones económicas, más allá del capital intelectual y del conocimiento con el que estas organizaciones son gestionadas (Nahapiet y Ghoshal, 1998). Por ello, Bucklin y Sengrupta (1993) sostienen que tanto el acceso a recursos como la adquisición de conocimiento son objetivos primordiales para la gestión del trabajo en red y su consecuente generación de capital social. La intensidad con que una empresa busca cumplir estos objetivos, a través de la interrelación organizacional, determina el nivel de capital social y de valor agregado deseado.

Las nociones de nivel de capital social y de valor agregado están altamente relacionadas pero son distintas, y el hecho de que una organización persiga como objetivo estratégico la consecución de una de ellas no significa que busque con ahínco la otra. La razón por la que esta situación sucede radica en la falta de compatibilidad percibida, por alguna de las partes involucradas, en materia de intercambio de recursos y conocimientos, al igual que en cierto nivel de desconfianza provocado por el miedo al oportunismo y a aprendizajes y ganancias asimétricas (Bucklin y Sengrupta, 1993).

\subsection{Las redes de contactos: esencia fundamental del networking}

Las redes de contactos pueden establecer muchas veces vías de comunicación vínculos relacionales - entre socios, clientes, proveedores, competidores y otros actores del entorno de cualquier emprendimiento, ya sea local, global o virtualmente. Estas actividades relacionales no son propias de un tipo específico de organización; sin embargo, han demostrado ser una herramienta importante para el desarrollo de pequeños y medianos emprendimientos. 
O’Donnell (2004) afirma que el auge de las interconexiones en red ha permitido a las empresas desarrollar ventajas competitivas y participar en proyectos económicos que no serían capaces de ejecutar individualmente. Al compartir recursos con otros emprendimientos se superan barreras de entrada a mercados que tradicionalmente han estado reservados a la participación exclusiva de la gran empresa. Dichas actividades podrían perfectamente confundirse con las nociones de cooperación, asociación e incluso clúster. La Organización para la Cooperación y el Desarrollo Económico (OCDE, 2000) define a las redes de contactos - en el ámbito económico - como la acción conjunta de un grupo de emprendedores que utilizan y comparten recursos para la consecución de proyectos comunes; representan conexiones relacionales propicias para la creación de estructuras más complejas de producción. Las redes pueden llevar a las empresas a compartir negocios, promociones, políticas de venta, fondos de capital, comercialización, información, servicios legales, proveedores, etc., en beneficio del cumplimiento de los objetivos estratégicos que se persigan, tanto individuales como colectivos.

En el campo del emprendimiento, las redes se definen como una interconexión mutua y opcional entre emprendedores basada en objetivos comunes que no necesariamente han de ser económicos. En muchas de las ocasiones, las interconexiones se promueven para reducir o eliminar amenazas compartidas del entorno empresarial, donde el desarrollo tecnológico y la fuerte influencia de las empresas multinacionales sirven de desencadenantes para la búsqueda de soluciones conjuntas.

El objetivo principal de la creación de conexiones relacionales es llegar a ser más competitivo a través de la reducción de costes de transacción y de producción, el acceso a información y tecnología actualizada, la creación de nuevas bases de datos, la generación de conocimiento de uso común, la puesta en marcha más ágil de productos y servicios novedosos, la posibilidad de penetrar en nuevos segmentos de mercado o el reparto del riesgo entre los miembros de la red.

Cooke (2002) propone que el entramado de interconexiones puede ser diferente en función del propósito para el que fueron creadas las redes, sugiriendo la siguiente clasificación:

- Redes informales; creadas en base a relaciones informales.

+ Redes formales: nacen fundamentalmente orientadas a ciertos objetivos individuales o comunes.

- Redes suaves: son redes abiertas que permiten un número ilimitado de miembros, por lo que suelen ser relativamente grandes. El intercambio de ideas y de información se genera sin restricciones. 
- Redes duras: son redes menos abiertas, establecidas bajo contrato y orientadas a llevar a cabo ciertas acciones en un horizonte temporal predeterminado.

- Redes verticales: se caracterizan por su fuerte interés por las redes que desarrollan sus clientes actuales o potenciales.

- Redes laterales: en términos generales están formadas por empresas altamente especializadas y de similar tamaño, usualmente complementarias, que comparten ciertas características con las redes formales o duras.

Dentro de este compendio clasificatorio, Hansen et alii (2000) consideran que son las estructuras informales - aquellas creadas en base a relaciones informales - las que producen el mayor desarrollo de redes de contactos en los procesos de incubación de negocios. Las organizaciones involucradas en la red pueden estar geográficamente concentradas en un entorno físico determinado, aunque a día de hoy las nuevas tecnologías también posibilitan que el entramado se desarrolle exclusivamente de manera virtual. Para Hämäläinen y Schienstock (2000), la infraestructura tecnológica representa la base de las complejas redes actuales, ya que los nuevos recursos tecnológicos modifican sustancialmente la concepción tradicional de las estructuras de redes.

\section{Diseño y metodología}

La metodología empleada en este trabajo parte de la identificación de una muestra válida. Al tratarse de un estudio exploratorio, con escasa existencia de antecedentes y trabajos similares, la muestra en principio debía comprender cierto número de emprendimientos asistidos por una misma unidad de incubación. La modalidad de asistencia podía ser, consiguientemente, de acompañamiento tanto externo como interno, y la gestora de incubación de empresas podía tener carácter privado, público o compartido. Resultaba recomendable investigar aquellos emprendimientos que estuviesen siendo gestionados geográficamente en la ciudad de Córdoba, conservando así el ánimo de conocer la realidad más próxima a nuestro entorno. El máximo responsable de cada una de estas empresas incubadas debía responder a un cuestionario dirigido a conocer el grado de interconexión en red de su organización, en relación con los diferentes agentes de interés.

Para la selección de la muestra se identificaron inicialmente, como posibles universos, cuatro instituciones cordobesas orientadas a brindar servicios de desarrollo empresarial a través de procesos de gestación de emprendimientos en espacios protegidos y/o bajo la modalidad de coaching externo. Estas fueron: IM- 
DEEC (Red de Viveros de Empresas), IDEA (Agencia de Innovación y Desarrollo de Andalucía), AJE (Asociación de Jóvenes Empresarios) y CADE (Centros de Apoyo al Desarrollo Empresarial). Por razones de discreción y de confidencialidad empresarial, solicitada por la unidad gestora finalmente escogida, nos debemos a la obligación de mencionar únicamente nuestro universo de selección y las características que justifican plenamente la selección final.

Todos los emprendimientos estudiados - 18 unidades de un vivero de 23 empresas - estaban siendo asistidos por la misma unidad gestora de incubación, de carácter público y perteneciente a la provincia de Córdoba, bajo la modalidad de acompañamiento o coaching externo. La muestra definitiva obtenida para el estudio exploratorio resultaba, por tanto, representativa y variada en términos estadísticos (78,3\% del total de emprendimientos posibles) y económicos: once de los emprendedores tenían como actividad principal la prestación de servicios $(61,1 \%)$, solo una unidad económica pertenecía al sector comercio $(5,6 \%)$, cuatro producían bienes no industriales $(22,2 \%)$ y las dos últimas podían ser consideradas como industriales $(11,1 \%)$.

El 21 de julio de 2011, aprovechando la invitación a una actividad destinada precisamente al desarrollo de redes relacionales denominada Jornada Migas de Verano, un equipo de 6 entrevistadores llevó a cabo las entrevistas personalizadas a los incubados, que dieron como resultado un total de 18 ejemplares válidos de las 23 empresas objeto de investigación.

La elaboración del cuestionario se fundamenta en dos premisas transversales: la selección de variables ajustadas a los objetivos del estudio y la identificación de los vínculos relacionales con sus correspondientes niveles de importancia y de frecuencia. A continuación se explica cada uno de estos puntos.

La elección de las variables se fundamentó en la intención implícita de aplicar la teoría de redes a la gestión de la incubación de negocios. Se hizo partícipes de dicho análisis, por tanto, a los actores o grupos de interés propios de cualquier emprendimiento: competidores, entidades financieras, aliados estratégicos, clientes, otros incubados, distribuidores, proveedores, otros proveedores, formación y trasferencia de conocimiento, promotores de innovación, asesoría administrativa, asesoría legal, servicios informáticos y equipos informáticos.

En cuanto a la selección de los tipos de preguntas, metodológicamente hablando, se utilizan tanto preguntas abiertas como cerradas. Para algunos casos se emplean escalas de Likert, con 6 alternativas de respuesta. Dado que el procesamiento de los datos obtenidos debe ser ordenado y graficado con la ayuda de soportes informáticos, el resultado de los cuestionarios se traslada al programa UCINET - en su versión 6.0-para su posterior desarrollo gráfico en NetDraw. 


\section{Principales resultados}

En la figura 1 se presentan las claves para la interpretación de los gráficos que muestran los vínculos relacionales que existen entre los nodos principales «incubados» $y$ los nodos secundarios stakeholders: competidores, entidades financieras, aliados estratégicos, clientes, otros incubados, etc. También se presentan las medidas cuantificables básicas del análisis de redes, como los niveles de importancia relacional, los niveles de frecuencia relacional o el origen de los vínculos.

En este sentido, las flechas indican los vínculos relacionales entre nodos, y el origen de la relación se expresa mediante la dirección de la flecha. Las formas de los nodos representan distintos rangos en los niveles de importancia relacional. Por último, los colores de los nodos simbolizan los valores asignados a la frecuencia relacional de los vínculos registrados.

Figura 1. Símbolos para la interpretación de los graficos «vínculos relacionales»

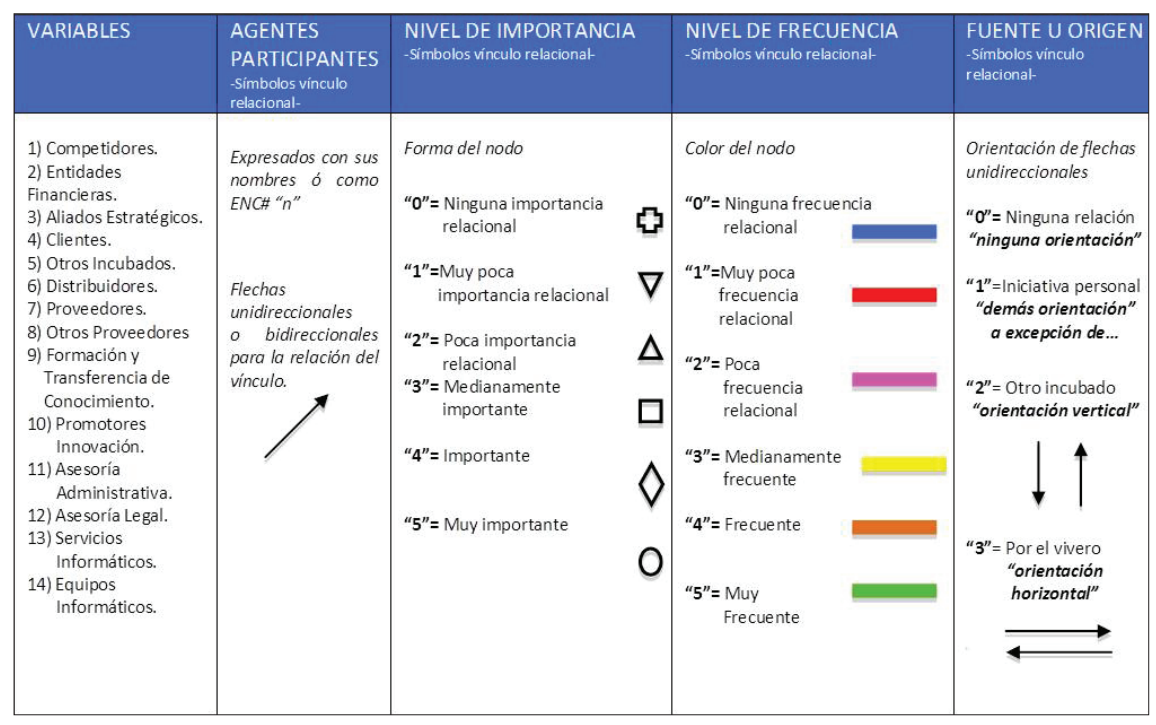

Elaboración propia. 
Figura 2. Vínculos relacionales «entidades financieras»

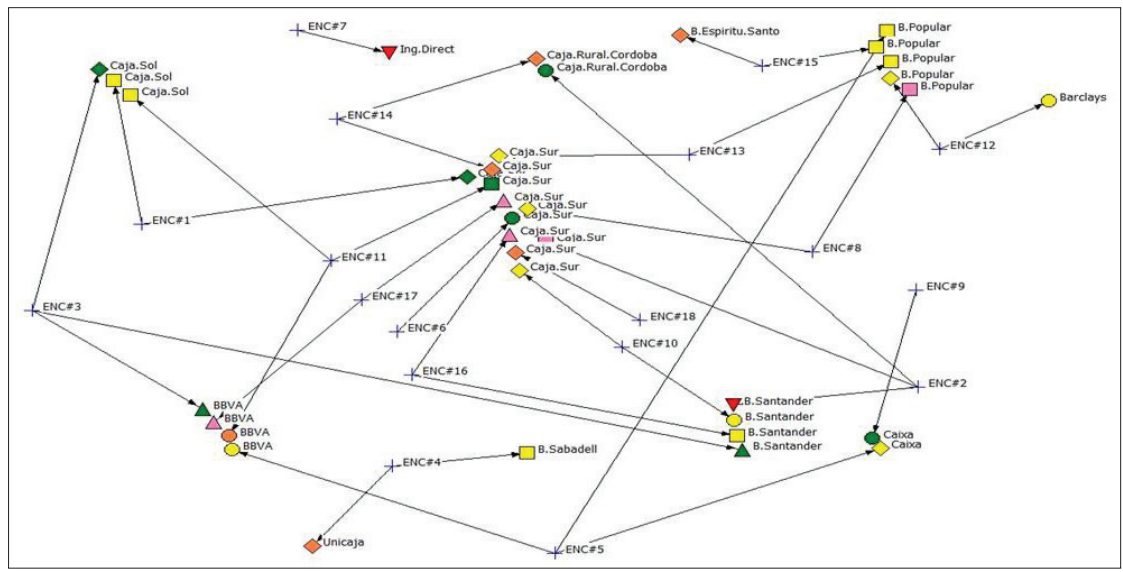

Elaboración propia.

Figura 3. Cuadro sinóptico de los resultados del estudio

\begin{tabular}{|c|c|c|c|c|c|c|c|c|}
\hline \multirow[t]{2}{*}{$\begin{array}{l}\text { Stakeholders/Medidas } \\
\text { cuantificables del } \\
\text { a nálisis de redes }\end{array}$} & \multirow[t]{2}{*}{$\begin{array}{l}\text { \# de inter- } \\
\text { relaciones }\end{array}$} & \multicolumn{2}{|c|}{$\begin{array}{l}\text { \#de } \\
\text { Inter- } \\
\text { relaciones } \\
\text { entre } \\
\text { incubados }\end{array}$} & \multirow[t]{2}{*}{$\begin{array}{l}\text { \# de inter- } \\
\text { relaciones } \\
\text { hacia } \\
\text { stakeholders }\end{array}$} & \multirow[t]{2}{*}{$\begin{array}{l}\text { \#de } \\
\text { núcleos } \\
\text { de } \\
\text { centralidad }\end{array}$} & \multirow[t]{2}{*}{$\begin{array}{c}\text { Rangos } \\
\text { predominantes } \\
\vdots \\
\text { importancia } \\
\text { de } \\
\text { conexiones } \\
\end{array}$} & \multirow[t]{2}{*}{$\begin{array}{l}\text { Rangos } \\
\text { predominantes: } \\
\text { frecuencia } \\
\text { de } \\
\text { conexiones }\end{array}$} & \multirow[t]{2}{*}{$\begin{array}{l}\text { Origen de } \\
\text { los vinculos } \\
\text { relacionales }\end{array}$} \\
\hline & & $\begin{array}{c}\text { Unt } \\
\text { direccional }\end{array}$ & $\begin{array}{c}\text { Bi- } \\
\text { direccional }\end{array}$ & & & & & \\
\hline A. Competidores & 39 & 0 & 0 & 39 & 0 & bajos/medios & $\begin{array}{c}\text { muy } \\
\text { bajos/medios }\end{array}$ & $\begin{array}{c}92,3 \% \\
\text { iniciativas } \\
\text { propias }\end{array}$ \\
\hline $\begin{array}{l}\text { B. Entidades } \\
\text { financieras }\end{array}$ & 36 & 0 & 0 & 36 & 4 & medios/altos & $\begin{array}{l}\text { medios/muy } \\
\text { altos }\end{array}$ & $\begin{array}{l}100 \% \\
\text { iniciativas } \\
\text { propias }\end{array}$ \\
\hline $\begin{array}{l}\text { C. Aliados } \\
\text { estratégicos }\end{array}$ & 27 & 0 & 0 & 27 & 1 & $\begin{array}{l}\text { medios/muy } \\
\text { altos }\end{array}$ & $\begin{array}{l}\text { mediosimuy } \\
\text { altos }\end{array}$ & $\begin{array}{l}100 \% \\
\text { iniciativas } \\
\text { propias }\end{array}$ \\
\hline D. Clientes & 36 & 0 & 0 & 36 & 2 & $\begin{array}{l}\text { altos/muy } \\
\text { altos }\end{array}$ & altosimuy altos & $\begin{array}{c}94,6 \% \\
\text { iniciativas } \\
\text { propias }\end{array}$ \\
\hline E. Otros incubados & 4 & 4 & 0 & 0 & 0 & $\begin{array}{c}\text { altosimuy } \\
\text { altos }\end{array}$ & altos/muy altos & $\begin{array}{l}100 \% \\
\text { iniciativas }\end{array}$ \\
\hline F. Distribuidores & 10 & 0 & 0 & 4 & 0 & medios & $\begin{array}{l}\text { medios/muy } \\
\text { altos }\end{array}$ & $\begin{array}{l}90 \% \text { iniciativas } \\
\text { propias }\end{array}$ \\
\hline G. Proveedores & 25 & 0 & 0 & 25 & 1 & $\begin{array}{l}\text { medios/muy } \\
\text { altos }\end{array}$ & $\begin{array}{l}\text { medios/muy } \\
\text { altos }\end{array}$ & $\begin{array}{l}100 \% \\
\text { iniciativas } \\
\text { propias }\end{array}$ \\
\hline H. Otros proveedores & 19 & 0 & 0 & 19 & 3 & medios/altos & mediosialtos & $\begin{array}{c}100 \% \\
\text { iniciativas } \\
\text { propias }\end{array}$ \\
\hline $\begin{array}{l}\text { I. Formacióny } \\
\text { transferencia } \\
\text { de conocimiento }\end{array}$ & 19 & 0 & 0 & 19 & 1 & medios/altos & medios & $\begin{array}{c}73,6 \% \\
\text { iniciativas } \\
\text { propias }\end{array}$ \\
\hline $\begin{array}{l}\text { J. Promotores de } \\
\text { innovación }\end{array}$ & 17 & 0 & 0 & 17 & 1 & medios & medios & $\begin{array}{l}73,7 \% \\
\text { iniciativas } \\
\text { propias }\end{array}$ \\
\hline $\begin{array}{l}\text { K. Asesoría } \\
\text { administrativa }\end{array}$ & 16 & 0 & 0 & 16 & 1 & medios/altos & mediosiattos & $\begin{array}{c}88,9 \% \\
\text { iniciativas } \\
\text { propias }\end{array}$ \\
\hline L. Asesoría legal & 18 & 0 & 0 & 18 & 1 & medios'altos & mediosialtos & $\begin{array}{c}100 \% \\
\text { iniciativas } \\
\text { propias }\end{array}$ \\
\hline $\begin{array}{l}\text { M. Servicios } \\
\text { informáticos }\end{array}$ & 15 & 0 & 0 & 15 & 0 & $\begin{array}{l}\text { medios/attos/ } \\
\text { muy altos }\end{array}$ & medios/altos & $\begin{array}{l}100 \% \\
\text { iniciativas } \\
\text { propias }\end{array}$ \\
\hline $\begin{array}{l}\text { N. Equipos } \\
\text { informáticos }\end{array}$ & 18 & 0 & 0 & 18 & 3 & medios/altos & mediosiattos & $\begin{array}{l}100 \% \\
\text { iniciativas } \\
\text { propias }\end{array}$ \\
\hline
\end{tabular}

Elaboración propia. 
La figura 2 ejemplifica el modelo de gráficas relacionales obtenidas: 14 en total. Este ejemplo, en particular, muestra los vínculos relacionales entre los incubados y los nodos secundarios que, para este caso en concreto, corresponden a las entidades financieras. En total se manifiestan 36 enlaces unidireccionales con estos agentes, pero ninguno entre diferentes incubados. Esto significa que ningún emprendedor acogido en el vivero realiza su actividad económica al margen de, como mínimo, una entidad financiera tradicional, que entre el resto de los incubados no existe ninguno que pueda facilitarle este servicio y que la mayoría de los nodos principales orientan sus relaciones hacia polos de centralidad.

Los niveles de importancia interrelacional para esta variable son porcentualmente más representativos para los puntos medios/altos y no extremos de la escala, las frecuencias en los vínculos relacionales predominantes presentan resultados medios o muy altos y el $100 \%$ de los vínculos relacionales con instituciones financieras surgen debido a «iniciativas propias».

La figura 3 muestra el comportamiento observado en las 14 gráficas obtenidas y resume los elementos explicativos con los que se han finalizado los dos párrafos anteriores para la totalidad de las gráficas. $\mathrm{Al}$ incluir la variable «competidores» en el análisis de los vínculos relacionales entre actores, el estudio sugiere que se debería tomar en cuenta anticipadamente si se ha de seleccionar una gestora de incubación que acompañe a empresas provenientes de distintos sectores económicos; si este es el caso, las interconexiones posiblemente tiendan a ser dispersas, unidireccionales y con agentes distintos a los incubados, debido a que las orientaciones empresariales de los emprendimientos son también diferentes $y$, en consecuencia, sus rivales en el mercado. En este contexto la centralidad de la red será reducida, resultando más que probable que los rangos asignados a la importancia y frecuencia de los vínculos se manifiesten con poca intensidad ya que, en relación con la competencia, lo que se desea es conocerla en profundidad y no establecer lazos estrechos, a menos que se transforme en un potencial aliado estratégico.

Este análisis es extrapolable a otras variables como «entidades financieras» $y$ «equipos informáticos»: los incubados posiblemente no tiendan a interrelacionarse-muy probablemente no serán organizaciones de la banca o ensambladores/comercializadores de portátiles-. Asimismo, cuanto más pequeño sea el radio de acción del vivero mayor tendencia habrá hacia cierta centralidad de agentes en la red - muchos vínculos y pocas entidades financieras - y los valores dados a la importancia y frecuencia de las interconexiones serán de medios a muy altos debido, posiblemente, a la importancia atribuida al capital y a las nuevas tecnologías en la gestión actual de emprendimientos. 
En el caso de las variables «aliados estratégicos», «clientes», «otros incubados»y distribuidores», el estudio parece indicar que, en dependencia de la densidad de la red, es muy probable que, debido a la diversidad de los emprendimientos y el área de acción de la incubadora, los vínculos relacionales se produzcan entre incubados en ambas direcciones o que confluyan hacia organizaciones de mayor influencia y envergadura en la comunidad. Se podrá esperar, por tanto, que los rangos asignados a la importancia y frecuencia de los vínculos sean altos o muy altos debido, precisamente, a la posible mayor influencia económica de estas organizaciones en sus emprendimientos. Por ello, es probable que, dado el limitado tamaño de la muestra para este estudio, ningún incubado haya referenciado a otro incubado como aliado estratégico o cliente.

De manera similar, el comportamiento de las interconexiones entre incubados y otros nodos presentes en los procesos de incubación - para las variables «proveedores», «otros proveedores», «asesoría administrativa», «asesoría legal» y «servicios informáticos»— posiblemente tienda a ser coincidente debido a la presencia o no de diversidad de emprendimientos, la densidad/tamaño de la red y el radio de influencia del vivero: cuanta más variedad de actividades económicas y de densidad/tamaño de la red se manifieste, mayor posibilidad de complementariedad entre los nodos habrá. Se podrán esperar valores de importancia y frecuencia relacional de medios a muy altos en correspondencia con la necesidad de abastecimiento de materia prima o de servicios.

Por otra parte, los vínculos relacionales pertenecientes a los componentes «formación y transferencia de conocimiento» $y$ «promotores de innovación» probablemente tiendan a confluir hacia un mismo nodo; en otras palabras, es posible que dichas provisiones de servicios sean brindadas por la misma unidad gestora del proceso de incubación o una entidad en concreto seleccionada por esta. En todo caso, los valores asignados a la importancia y frecuencia de las interconexiones probablemente sean de altos a muy altos, pues la búsqueda de conocimiento y la generación de innovación son dos de las razones principales que mueven a los emprendedores a participar en los procesos de incubación.

\section{Conclusiones}

Sobre la base de una muestra de 18 emprendimientos incubados bajo la modalidad de acompañamiento o coaching externo, pertenecientes a una unidad de incubación del sector público, la presente investigación explora, por un lado, las posibilidades de aplicar la teoría de redes al estudio de la gestión de viveros de emprendimientos $y$, por otro, las características de los vínculos relacionales e in- 
terconexiones generadas entre incubados como producto de la gestión de networking en los procesos de incubación.

Los hallazgos obtenidos indican que el comportamiento de los vínculos relacionales es muy variado en las catorce matrices resultantes, aunque apenas se manifiestan interconexiones entre los incubados. Esta baja densidad de la red sugiere que ninguno de los incubados actúa como grupo de interés directo para otros, desaprovechando así las posibles sinergias entre empresas que comparten una situación parecida.

El hecho de no contar con una población integralmente interconectada conlleva una serie de limitaciones para la posible aplicación del análisis de redes en términos holísticos, con la aplicación de medidas cuantificables de mayor profundidad y significancia. Sin embargo, el estudio sugiere algunas recomendaciones que tener en cuenta para futuras investigaciones en este campo, que permitan obtener magnitudes medibles de mayor peso crítico.

La primera consideración que evaluar es si se debe o no seleccionar viveros de empresas que acompañen a sus incubados bajo el régimen de coaching externo o interno. Una convivencia muy estrecha no significa necesariamente que existan vínculos relacionales entre incubados muy fuertes (redes densas). La decisión sobre este asunto puede dar lugar al diseño de estudios comparativos que permitan profundizar sobre el tema.

Otro elemento que considerar es el tamaño de la unidad gestora del proceso de incubación. La experiencia de este trabajo sugiere que el vivero investigado debe integrar una muestra de empresas algo más amplia que la actual, lo que posiblemente implique a gestoras de incubación de negocios altamente relacionadas con otras gestoras y que integren a emprendimientos variados. Sin embargo, existe también la posibilidad de estudiar viveros especializados en sectores concretos.

Es de igual forma recomendable incluir en análisis futuros la variable tiempo de acompañamiento con el que una incubadora asiste a los emprendedores de su red, ya que parece lógico que a mayor tiempo se produzcan mayores vínculos relacionales entre incubados.

Finalmente habría que indagar sobre el grado de integración de la gestión del networking dentro de la estrategia general de la incubadora; es decir, ¿̧hasta qué nivel permanece institucionalizado el networking en las tareas diarias? La obtención de datos acerca del origen de los vínculos es importante, pero también se precisa de información complementaria que indique el compromiso organizacional real del vivero: grado de recursos asignados a la gestión del networking, tiempo invertido entre las partes, espacios disponibles, fondos destinados a estas actividades, etc. 


\section{Bibliografía}

Aerts, K.; Matthyssens, P. y Vandenbempt, K. (2007). «Critical Role and Screening Practices of European Business Incubators». Technovation, 27: 254-267.

Albert, P. y Gaynor, L. (2001). Incubators: Growing up, Moving out -a Review of the Literature. Sophia Antipolis: Chair of High Tech Entrepreneurship, CERAM.

Allen, D. N. y MacCluskey, R. (1990). «Structure, policy, services, and performance in the business incubator industry». Entrepreneurship, Theory and Practice, 15 (2): 61-77.

Bailey, E. E. y Friedlander, A. F. (1982). «Market structure and multiproduct industries». Journal of Economics Literature, 20 (3): 1024-1048.

Bollingtoft, A. y Ulhoi, J. (2005). «The network business incubator leveraging entrepreneurial agency?». Journal of Business Venturing, 20: 265290.

Bolino, M. C. et alii (2002). «Citizenship behavior and the creation of social capital in organizations». Academy of Management Review, 27: 505-522.

Bucklin, L. P. y Sengrupta, S. (1993). «Organizing successful co-marketing alliances». Journal of Marketing, 57 (2): 32-46.

Burt, R. S. (1992). Structural Holes: The Social Structure of Competition. Cambridge: Harvard University Press.

Coleman, J. S. (1988). «Social capital in the creation of human capital». American Journal of Sociology, 94: 95-120. Supplement: Organizations and Institutions: Sociological and Economic Approaches to the analysis of Social Structure.

Coокe, P. (2002). Knowledge Economies: Clusters, Learning and Cooperative Advantage. London: Routledge.

Dyer, J. H. y Singh, H (1998). «The relational view: cooperative strategy and sources of interorganizational competitive advantage». Academy of Management Review, 23 (4): 660-679.

Eтzкоwiтz, H. (2002). «Incubation of incubators: innovation as a triple helix of university-industry-government networks». Science and Public Policy, 29 (2): 115-128.

European Comission (2002). Benchmarking of business incubators, Brussels. Ford, D., Gadde, L.; Hakansson, H.; Lundgren, A.; Turnbull, P; Wilson, D., y Snehota, I. (1998). En: Ford, D. (ed.). Managing Business Relationships. Chichester: Wiley. 
Freel, M. S. (2003). «Sectoral patterns of small firms innovation, networking and proximity». Research Policy, 32 (5): 751-770.

Hackett, S. M. y Dilts, D. M. (2004). «A systematic review of business incubation research». The Journal of Technology Transfer, Vol. 29 (1):55-82.

Hansen, M. et alii (2000). "Networked incubators: hothouse of the new economy». Harvard Business Review, Sept-Oct.: 74-84.

Hämäläinen, T. J. y Schienstock, G. (2000). Innovation Networks and Network Policies. Report for OECD Research Project on National Innovation Systems: Workshops and Meetings of the Focus Group on Innovative Firms and Networks.

Hughes, M.; Ireland, R.D., y Morgan, R. (2007). «Stimulating dynamic value: social capital and business incubation as a pathway to competitive success». Long Range Planning, 40: 154-177.

Ireland, R. D. et alii (2002). «Alliance management as a source of competitive advantage». Journal of Management, 28: 413-446.

Johannisson, B. (1988). «Business formation -a network approach». Scandinavian Journal of Management, 4 (3-4): 83-99.

Knopp, L. (2007). 2006 State of the Business Incubation Industry. Athens: NBIA Publications.

Larson, A. (1991). "Partner network: leveraging external ties to improve entrepreneurial performance». Journal of Business Venturing, 6: 173-188.

Lin, N. (2001). Social Capital: A Theory of Social Structure and Action. New York: Cambridge University Press.

Lyons, T. S. (2000). «Building Social Capital for Sustainable Enterprise Development in Country Towns and Regions: Successful Practices from the United States». Artículo presentado durante la 1. ${ }^{a}$ Conferencia Nacional sobre el Futuro de las Ciudades en Australia. LaTrobe University; Center for Sustainable Regional Communities, Australia. June 29-30.

Nahapiet, J. y Ghoshal, S. (1998). «Social capital, intellectual capital, and the organizational advantage». Academy of Management Review, 23 (2): 242-266.

O'Donnell, A. (2004). "The Nature of Networking in Small Firms». Qualitative Market Research, 7 (3): 206-217.

OECD (2000). Enhancing the competitiveness of SMEs in the Global Economy. Local Partnership, Clusters and SME Globalisation. Conference for Ministers responsible for SMEs and Industry Ministers; Bolonia, Italy; 14-15 June.

Panzar, J. C. y Willig, R.D. (1981). «Economics of scope». American Economic Review, 71 (2): 268-272. 
Schumpeter, J. A. (1942). Capitalism, Socialism and Democracy. New York: Harper y Brothers.

Smilor, R. W. y Gill, M.D. (1986). The New Business Incubator. Linking Talent, Technology, Capital, and Know-How. Massachusetts, Toronto: Lexington Books.

Tse, E. (2002). «Grabber-holder dynamics and network effects in technology innovation». Journal of Economics Dynamics and Control, 26 (9-10): 17211738.

Uzzi, B. (1996). "The sources and consequences of embeddedness for the economic performance of organizations: the network effect». American Sociological Review, 61 (4): 674-698.

Williamson, O. E. (1975). Market and Hierarchies: Analysis and Antitrust Implications. New York: The Free Press. 